\title{
Minimising radiation exposure in catheter ablation of ventricular arrhythmias
}

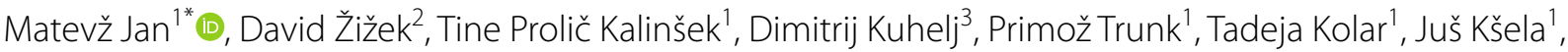 \\ Martin Rauber ${ }^{2}$ and Mehmet Yazici ${ }^{1}$
}

\begin{abstract}
Background: Conventional fluoroscopy guided catheter ablation (CA) is an established treatment option for ventricular arrhythmias (VAs). However, with the complex nature of most procedures, patients and staff bare an increased radiation exposure. Near-zero or zero-fluoroscopy CA is an alternative method which could substantially reduce or even eliminate the radiation dose. Our aim was to analyse procedural outcomes with fluoroscopy minimising approach for treatment of VAs in patients with structurally normal hearts (SNH) and structural heart disease (SHD).
\end{abstract}

Methods: Fifty-two (age 53.4 17.8 years, 38 male, 14 female) consecutive patients who underwent CA of VAs in our institution between May 2018 and December 2019 were included. Procedures were performed primarily with the aid of the three-dimensional electro-anatomical mapping system and intra-cardiac echocardiography. Fluoroscopy was considered only in left ventricular (LV) summit mapping for coronary angiography and when epicardial approach was planned. Acute and long-term procedural outcomes were analysed.

Results: Sixty CA procedures were performed. Twenty-five patients had SHD-related VAs (Group 1) and 27 patients had SNH (Group 2). While Group 1 had significantly higher total procedural time (256.9 \pm 71.7 vs $123.6 \pm 42.2 \mathrm{~min}$; $p<0.001)$ compared to Group 2, overall procedural success rate [77.4\% (24/31) vs 89.7\% (26/29); $p=0.20)$ ] and recurrence rate after the first procedure [8/25, (32\%) vs $8 / 27,(29.6 \%) ; p=0.85]$ were similar in both groups. Fluoroscopy was used in 3 procedures in Group 1 where epicardial approach was needed and in 4 procedures in Group 2 where LV summit VAs were ablated. Overall procedure-related major complication rate was $5 \%$.

Conclusions: Fluoroscopy minimising approach for CA of VAs is feasible and safe in patients with SHD and SNH. Fluoroscopy could not be completely abolished in VAs with epicardial and LV summit substrate location.

Keywords: Ventricular arrhythmia, Radiation exposure, Catheter ablation, Zero-fluoroscopy

\section{Background}

Catheter ablation (CA) guided by conventional fluoroscopy (CF) is an established treatment option for ventricular arrhythmias (VAs) [1]. However, the use of fluoroscopy exposes patients and medical staff to potentially harmful stochastic and deterministic effects of ionising radiation [2-4]. This risk is significantly increased

\footnotetext{
*Correspondence: matevz.jan@kclj.si

${ }^{1}$ Cardiovascular Surgery Department, University Medical Centre

Ljubljana, Zaloška 7, 1000 Ljubljana, Slovenia

Full list of author information is available at the end of the article
}

in long-lasting, complex, and multiple ablation procedures like CA of atrial fibrillation (AF) and VAs, with $60 \mathrm{~min}$ of fluoroscopy resulting in a lifetime risk of $0.07 \%$ for women and $0.1 \%$ for men for fatal malignancy [5-7]. Reduction of radiation exposure during CA procedures with employing the ALARA (as low as reasonably achievable) principles is an already established routine in electrophysiology laboratories [8].

In recent years, reports of near-zero (NZF) and zerofluoroscopy (ZF) procedures show that it is possible to significantly reduce or even eliminate radiation 
exposure in most CA procedures. Fluoroscopy minimising approaches utilising three-dimensional (3D) electro-anatomical mapping (EAM) systems have been frequently used in children and adults with supra-ventricular tachycardias (SVTs) and in adults with AF [918]. However, reports of NZF or ZF approaches in CA of different forms of VAs are relatively scarce [19-21].

Our aim was to analyse procedural outcomes with fluoroscopy minimising approach for treatment of VAs in patients with structurally normal hearts $(\mathrm{SNH})$ and structural heart disease (SHD).

\section{Methods}

\section{Study population}

Consecutive patients who underwent CA of VAs in our institution between May 2018 and December 2019 were included. In general, patients with $\mathrm{SNH}$ and idiopathic VAs, as well as patients with ventricular tachycardias (VT) resulting from SHD were included.

Indications to undergo CA were followed according to the published guidelines and consensus documents [1]. Included patients with SNH had an ECG documentation of ventricular ectopy, with an estimated daily ectopy burden of at least $10 \%$, or tachycardia that were causing either severe symptoms unresponsive to anti-arrhythmic drugs (AADs), or presumed tachycardia-induced cardiomyopathy. All included patients with SHD had a documented sustained VA either with 12-lead ECG or a cardiac implantable electronic device (CIED).

\section{Pre-procedural management}

Trans-thoracic echocardiogram was performed in all patients before the CA procedure. Pre-procedural computed tomography (CT) or magnetic resonance imaging (MRI) were accessible in most patients, however, images obtained were not used to create three-dimensional reconstructions for procedural purposes. All patients with a suspected SHD had pre-procedural assessment of coronary anatomy with invasive coronary angiography or CT angiography. Combined results of imaging studies were used to assess the presence of SHD.. Basic laboratory blood tests, clinical examination and CIED interrogations were also performed in all patients.

\section{Procedural setup}

Procedures were preferably performed in conscious sedation, except for patients undergoing epicardial approach in whom general anaesthesia was used. Femoral vein and artery punctures were performed to access the heart. A 3D EAM system $\left(\right.$ Carto $^{\circledR}$, Biosense Webster, Irvine, California, USA or NavX ${ }^{\mathrm{TM}}$ Precision $^{\mathrm{TM}}$, Abbott, Abbott Park, Illinois, USA) and intra-cardiac echocardiography (ICE) (Acuson AcuNav ${ }^{\mathrm{TM}}$, Biosense Webster, Irvine, California,
USA) were routinely used simultaneously. In patients with left-sided arrhythmias, we generally used the antegrade trans-septal approach, except when mapping and ablation in the aortic root were needed in which case a retrograde trans-aortic approach was used. A steerable long sheath (Agilis ${ }^{\mathrm{TM}}$, LRG Curl, Abbott, Abbott Park, Illinois, USA) was used to improve catheter stability in trans-septal approach. Typically, uni-directional irrigated tip radio-frequency (RF) ablation catheters with (Thermocool Smarttouch ${ }^{\circledR}$ and Thermocool Smarttouch ${ }^{\circledR}$ SF, both Biosense Webster, Irvine, California, USA) and without (FlexAbility ${ }^{\mathrm{TM}}$, Abbott, Abbott Park, Illinois, USA) the ability of contact force sensing were utilised. In patients with SHD and suspected substantial arrhythmogenic substrate we used a multipolar catheter enabling high-density mapping (PentaRay ${ }^{\circledR}$, Biosense Webster, Irvine, California, USA).

\section{Procedural workflow}

Once all catheters and the ICE probe were inserted, we followed two basic protocols depending on the type of arrhythmia.

1. In patients with a SHD and a recorded VA, the first step was VT induction with programmed ventricular stimulation. The next step was substrate mapping with the ablation catheter and later detailed mapping with the multipolar mapping catheter. Anatomical and voltage maps of the chamber of interest were created with the 3D EAM system and all electrograms with characteristics of local abnormal ventricular activity (LAVA) [22] and obvious late and diastolic electrical potentials (LP) were tagged [23]. Additionally, the areas of the substrate with suspected LAVA and without obvious LP were mapped during decremental RV pacing to reveal hidden slow conduction, which was also tagged [24]. Substrate mapping was combined with limited pace-mapping to reveal the exit point of the induced VT, with adjacent low voltage areas thoroughly mapped. Finally, all areas with late potentials and hidden slow conduction were extensively ablated using the described irrigated catheters with power-controlled settings of 30-45 W for at least $40 \mathrm{~s}$ or until the disappearance or at least attenuation of the local electrocardiogram. A re-mapping of the ablated parts of the substrate and surrounding areas with the multipolar mapping catheter was routinely performed and additional ablations were done if needed to achieve complete substrate modification, i.e. absence of local electrical activity. Endpoints of the procedure were substrate modification and non-inducibility of VT with programmed ventricular stimulation with up to four 
delivered extrasystoles combined with isoproterenol infusion.

2. In patients with presumably idiopathic VAs only very partial anatomical and voltage maps were created with the 3D EAM. Typically, our strategy relied on activation mapping of the present ventricular ectopy or VT. Pace-mapping was also used to complement activation mapping when needed. The mapped origin of the arrhythmia was ablated with similar settings and parameters as described in patients with SHD. The endpoint was termination of the ectopy or tachycardia. An additional endpoint was the absence and non-inducibility of VAs with fast and programmed ventricular stimulation, also after infusion of isoproterenol.

\section{Epicardial approach}

When epicardial mapping was needed a steerable long sheath (Agilis EPI ${ }^{\mathrm{TM}}$, Abbott, Abbott Park, Illinois, USA) was inserted over the J-tip guide-wire through surgically prepared sub-xiphoid epicardial approach.

\section{Fluoroscopy minimising approach}

Procedures were generally performed without any fluoroscopy and only with the use of the 3D EAM system and ICE. Fluoroscopy was only considered in two sets of cases: 1 . In LV summit mapping for coronary angiography to avoid RF delivery in close vicinity of a coronary arteries. 2. In procedures where the epicardial approach for mapping and ablation was planned. Fluoroscopy time and dose area product (DAP) were measured and reported for each procedure.

\section{The use of intra-cardiac echocardiography}

ICE was used in conjunction with the 3D EAM system in all procedures. ICE had four basic roles:

1. Providing guidance of guide-wire, long sheath, and trans-septal needle during trans-septal puncture (TSP) as previously described [25].

2. Providing additional imaging information about heart anatomy relevant for accurate and effective endocardial mapping and ablation. Examples include: achieving catheter stability at certain regions of the ventricles such as papillary muscles and moderator band; observing direction and position of the long sheath and catheter loops in cases of mapping and ablation at the base of the ventricles.

3. Replacing coronary angiography for visualisation of coronary artery ostia during ablation in the region of the sinuses of Valsalva.
4. Timely detection of possible complications such as sudden tissue whitening preceding "steam pops", pericardial effusion, potential thrombotic masses in the heart cavities or on sheaths and catheters inserted into the heart, sudden deterioration of systolic function of the ventricles.

\section{Definition of procedural parameters, procedural success, complications and follow-up}

The procedural duration (total procedural time, TPT) was defined as the time interval from the placement of the venous and arterial sheaths to their removal at the end of the ablation procedure. In the case of a surgical sub-xiphoid epicardial approach time measurement was started with skin incision. Ablation time [time spent delivering radio-frequency (RF) energy to the tissue)] and the number of ablations were automatically recorded by the intra-cardiac electrogram registration and analysis system. Procedures were considered successful if predefined endpoints were reached. Overall procedural success rate (OSR) was defined as procedural success of combined first and repeat procedures for treated VA. Recurrence rate (RR) was defined as recurrence of the treated VA after the first procedure. Any early (during the same hospital stay) and late complications were reported as complication rate $(\mathrm{CR})$, calculated as percentage of procedures. Major complications were all adverse events resulting in procedure termination, prolonged hospital stay, long-term disability, and any additional intervention and bleeding requiring transfusion.

Patients were followed clinically and with the 12-lead ECG recordings 3 months after the procedure and every 6 months thereafter. Patients with a CIED had its telemetry examined at every visit for possible episodes of VT, appropriate and inappropriate interventions, such as anti-tachycardia pacing (ATP), direct current cardioversions, and defibrillations. In patients treated for sustained VT, it's recording during the follow-up (ECG or CIED tracing) was regarded as a recurrence. In patients treated for ventricular ectopy, 24-h holter recordings were typically used to assess the daily VAs burden at least one month after the ablation procedure. If the VAs burden was comparable to the one expected in the normal population, patients were considered to be cured and without recurrence. Procedures were considered successful if expected endpoints were reached. Any recurrences were used to calculate the recurrence rate (RR).

\section{Statistical analysis}

The statistical analyses were carried out using the IBM SPSS software version 24.0 for Windows (IBM Corporation, Armonk, NY, USA). Continuous variables were 
presented as mean \pm standard deviation (SD) while categorical variables were presented as number and percentage. Differences between groups were analysed using unpaired Student's t-test for numeric variables and Pearson Chi-square test and Fisher exact test for attributive variables. Multiple group comparisons were performed with one-way ANOVA. $p$ value was considered significant when it was less than 0.05 .

\section{Results}

Fifty-two (age $53.4 \pm 17.8$ years, 38 males, 14 females) patients had $60 \mathrm{CA}$ procedures for treatment of VAs. Twenty-five patients had SHD-related VAs (Group 1), among which 19 patients had VT due to ischaemic cardiomyopathy (ICM), 6 had ventricular arrhythmia/tachycardia due to non-ischaemic cardiomyopathy (NICM) and 1 had a combination of congenital heart disease and ischaemic heart disease (IHD). There were 27 patients with SNH and idiopathic VAs (Group 2). Detailed baseline demographic and clinical characteristics are presented in Table 1.

\section{Procedural parameters}

Group 1 had significantly higher TPT compared to group $2(256.9 \pm 71.7$ and $123.6 \pm 42.2 \mathrm{~min} ; p<0.001)$ (Table 2). Overall procedural success rate (OSR), including the repeat procedures, was $83 \%(50 / 60)$ for the entire study population. OSR was similar in both groups [77.4\% $(24 / 31)$ and $89.7 \%(26 / 29) ; p=0.20)]$.

\section{Procedural outcomes}

During the follow-up period of $255 \pm 170$ days, there were 16 recurrences after the first CA procedure $(16 / 52$, RR 30.7\%). RR after the first procedure was similar in both groups [8/25 (32\%) and 8/27 (29.6\%); $p=0.85]$. Detailed information on procedural findings and parameters are presented in Table 2.

All idiopathic VAs originating from the RV (12 from RVOT) were successfully ablated $(16 / 16,100 \%)$ and there were no arrhythmia recurrences during follow-up. In 9 patients with VAs originating from LV 77.7\% (7/9) procedural success rate was recorded and there were 2 recurrences $(22.2 \%)$ after the initial ablation. In patients with NICM and ICM, OSR [respectively; $62.5 \%(5 / 8)$ and $82.6 \%(19 / 23) ; p=0.18)]$ and RR after the first ablation [respectively; $33.3 \%(2 / 6)$ and $26.3 \%(5 / 19) ; p=0.27)$ ] were similar.

Patients with failed procedures in Group 1 included one patient with IHD (intramural substrate) that had 3 procedures including epicardial mapping and ablation and one patient with NICM (post-myocarditis aetiology of intramural ectopy substrate) that had two failed endocardial procedures. In both patients arrhythmia occurrence was successfully controlled with amiodarone.

Table 1 Demographic and clinical characteristics of the study population.

\begin{tabular}{|c|c|c|c|}
\hline & $\begin{array}{l}\text { All } \\
(n=52)\end{array}$ & $\begin{array}{l}\text { Group } 1 \\
(n=25)\end{array}$ & $\begin{array}{l}\text { Group 2 } \\
(n=27)\end{array}$ \\
\hline Age (years) & $53.4 \pm 17.8$ & $62.8 \pm 12.5$ & $44.7 \pm 17.7^{*}$ \\
\hline Male gender [number (\%)] & $38(73.1)$ & $24(96 \%)$ & $14(51.9)^{*}$ \\
\hline Body Mass Index (kg/m²) & $25.6 \pm 5.2$ & $27.1 \pm 3.8$ & $24.2 \pm 6.0^{* *}$ \\
\hline Arterial hypertension [number (\%)] & $27(51.9)$ & $19(76)$ & $8(29.6)$ \\
\hline Hyperlipidemia [number (\%)] & $27(51.9)$ & $19(76)$ & $8(29.6)$ \\
\hline Diabetes mellitus [number (\%)] & $5(9.6)$ & $3(12)$ & $2(7.4)$ \\
\hline Ischaemic cardiomyopathy [number (\%)] & $19(36.5)$ & $19(76)$ & 0 \\
\hline Non-ischaemic cardiomyopathy [number (\%)] & $5(9.6)$ & $5(20)$ & 0 \\
\hline Congenital heart disease [number (\%)] & $1(1.9)$ & $1(4)$ & 0 \\
\hline $\operatorname{LVEF}(\%)$ & $49.5 \pm 13.1$ & $40.8 \pm 10.4$ & $57.5 \pm 9.7^{*}$ \\
\hline LVEDV (ml) & $154.5 \pm 62.4$ & $194.7 \pm 64.3$ & $117.1 \pm 28.5^{*}$ \\
\hline RV dysfunction [number (\%)] & $5(9.6)$ & $4(16)$ & $1(3.7)$ \\
\hline CIED present[number (\%)] & $16(30.7)$ & $16(64)$ & 0 \\
\hline AAD non-amiodarone [number (\%)] & $34(65.3)$ & $21(84)$ & $13(48.1)$ \\
\hline Amiodarone [number (\%)] & $9(17.3)$ & $7(28)$ & $2(7.4)$ \\
\hline Sustained VT [number (\%)] & $15(28.8)$ & $10(40)$ & $5(18.5)$ \\
\hline Electrical storm [number (\%)] & $8(15.4)$ & $8(32)$ & 0 \\
\hline Tachycardia induced cardiomyopathy [number (\%)] & $4(7.6)$ & 0 & $4(14.8)$ \\
\hline
\end{tabular}

${ }^{*} p<0.001$; group I versus Group II, ${ }^{* *} p<0.05$; group I versus Group II

$\angle V E F$ left ventricular ejection fraction, $L V E D V$ left ventricular end-diastolic volume, $R V$ right ventricular, $C E I D$ cardiac implantable electronic device, $A A D$ anti-arrhythmic drugs, VT ventricular tachycardia 
Table 2 Procedural characteristics

\begin{tabular}{lll}
\hline & $\begin{array}{l}\text { Group 1 } \\
\text { (n=31) }\end{array}$ & $\begin{array}{c}\text { Group 2 } \\
\text { (n= 29) }\end{array}$ \\
\hline Septal substrate related VT [number (\%)] & $8(25.8)$ & - \\
Epicardial substrate related VT [number (\%)] & $6(19.4)$ & - \\
Outflow tract ectopy/NT [number (\%)] & - & $18(62.1)$ \\
Fascicular VT [number (\%)] & - & $2(6.9)$ \\
Moderator band ectopyNT [number (\%)] & - & $2(6.9)$ \\
Postero-septal process ectopy/NT [number (\%)] & - & $4(13.8)$ \\
Papillary muscle ectopy/NT [number (\%)] & - & $1(3.4)$ \\
Other substrate/origin locations [number (\%)] & $28(90.3)$ & $4(13.8)$ \\
Total procedural time (min) & $256.9 \pm 71.7$ & $123.6 \pm 42.2^{*}$ \\
Number of ablations (number) & $80.9 \pm 40.5$ & $15.4 \pm 8.6^{*}$ \\
Total ablation time (seconds) & $3240 \pm 1690$ & $537 \pm 357^{*}$ \\
Trans-septal punctures [number (\%)] & $28(90.3)$ & $8(27.6)^{*}$ \\
Epicardial mapping and ablation [number (\%)] & $3(9.7)$ & $4(13.8)$ \\
Procedures with high density mapping [number (\%)] & $25(80.6)$ & $4(13.8)^{*}$ \\
Procedures with general anaesthesia [number (\%)] & $6(19.4)$ & $4(13.8)$ \\
Overall Procedural Success Rate [number (\%)] & $24(77.4)$ & $26(89.7)$ \\
Repeat procedures [number (\%)] & $6(19.4)$ & $2(6.9)$ \\
Procedures with fluoroscopy [number (\%)] & $3(9.7)$ & $4(13.8)$ \\
Fluoroscopy time (min) & $21.2 \pm 5$ & $8.3 \pm 4$ \\
DAP per procedure [Gycm² (SD)], if fluoroscopy used & $48.6 \pm 23(3$ procedures) & $25.4 \pm 14(4$ procedures) \\
\hline VT v & & \\
\end{tabular}

$V T$ ventricular tachycardia, DAP dose area product, $S D$ standard deviation ${ }^{*} p<0.001$; group I versus Group II

There were two ICM patients (epicardial origin of arrhythmia) with one failed endocardial procedure. After bisoprolol up-titration they did not require repeat procedures. Similarly, patients with failed procedures in Group 2 included one patient with LV-summit ectopy that was successfully ablated after repeat procedure; one patient with ectopy from LV postero-septal process that was successfully ablated at the repeat procedure; one patient with ectopy from the commissure between the left and right aortic cusps that refused repeat ablation and whose ectopy was apparently sufficiently suppressed to enable normalisation of previously reduced systolic function of LV during follow-up. One patient with ICM that had CA due to a VT storm shortly after emergency heart surgery died approximately one month after the ablation procedure because of heart surgery related complications.

\section{Use of fluoroscopy}

Three procedures in Group $1(3 / 31,10 \%)$ required additional X-ray fluoroscopy that was used for $21.2 \pm 5 \mathrm{~min}$ with DAP of $48.6 \pm 32 \mathrm{Gycm} 2$. In all three procedures endocardial and epicardial ablation were performed (Fig. 1). Two procedures were repeat procedures in patients with ICM. One procedure was a first-time CA in a patient with arrhythmogenic cardiomyopathy of RV and
LV. Procedural success was achieved in two $(2 / 3,66 \%)$ epicardial procedures. There were no arrhythmia recurrences, even in the procedure that was considered unsuccessful, however, this patient was eventually treated with amiodarone. Four procedures $(4 / 29,14 \%)$ in Group 2 required X-ray fluoroscopy that was used for $8.3 \pm 4 \mathrm{~min}$ with DAP of $25.4 \pm 14 \mathrm{Gycm} 2$. In all four procedures LVsummit area was the origin of VA and epicardial mapping and ablation was needed in the great cardiac vein (Fig. 2). Additionally, coronary angiography had to be performed to avoid RF delivery close to coronary artery. All four $(4 / 4,100 \%)$ procedures were acutely successful with one recurrence during follow-up. Four VA ablation procedures in Group 2 were successfully performed in paediatric patients without the use of X-ray fluoroscopy.

\section{Procedural adverse events}

As shown in Table 3 there were overall $3(3 / 60,5 \%)$ major complications related to the procedures. One patient with an ICM had perforation of RV during an attempted surgical approach to the epicardium. The perforation was also treated surgically. One patient had a late pericardial tamponade occurring 1 week after successful ablation of idiopathic RVOT ectopy which required percutaneous 


\section{(See figure on next page.)}

Fig. 1 Epicardial ablation. (A, B) Both present a modified left lateral view of a partial three-dimensional (3D) electro-anatomical mapping (EAM) voltage map of the epicardial part of the left ventricle (LV) with purple dots representing local abnormal ventricular activity (LAVA) sites inside and on the border of the low voltage area and red dots representing eventual ablation lesions. The white arrows mark the approximate location of the recorded LAVA as shown on intra-cardiac electrogram recordings made with multipolar mapping catheter presented on (C) (Pentaray 1-20) and with ablation catheter presented on (D) (ABLD and ABLP). The white asterisks mark the recorded isolated diastolic potentials at one of the eventual ablation sites near the course of the left circumflex artery $(L C X)$ at the base of the epicardial side of the left ventricle. Before ablation at that site, selective left coronary angiography in a modified right anterior oblique view was performed as shown on (E, F). Red arrow on (E) points at the tip of the ablation catheter touching the proximal part of the LCX in which case the catheter was slightly withdrawn to the site marked with the white arrow on (F) showing the tip at the safe distance from the LCX at the location of the eventual successful ablation. CSD-CSP intra-cardiac electrograms recorded with a 10-polar diagnostic catheter in the right ventricle; I, II, aVF and V1 surface electrograms

drainage. One patient with an ICM had pseudo-aneurysm of the left femoral artery that was treated surgically.

\section{Discussion}

The main findings of our study show that fluoroscopy minimising approach for CA procedures can be safely and efficiently performed in most patients with VAs. Procedural outcomes of this approach were similar in patients with SHD and SNH. Fluoroscopy was only used in 3 SHD patients with epicardial substrate and in $4 \mathrm{SNH}$ cases with LV summit substrate where intra-procedural coronary angiography was needed to avoid RF delivery in close vicinity of coronary arteries.

The main findings of our study are consistent with the results of earlier studies on efficacy and safety of CFbased CA in patients with and without SHD [26-28]. In a prospective study involving 227 adults with SHD (63 NICM and 164 ICM) and VT, Dinov et al. [26] demonstrated that the non-inducibility of VT was achieved in $66.7 \%$ of NICM and in $77.4 \%$ of ICM patients that were ablated with the aid of CF combined with the 3D EAM system. Complication rate in both groups was similar (11.1\% for both groups), however, higher compared to ours $(8.6 \%$ for ICM and $0 \%$ for NICM). In addition, reported VT-free survival rates $(40.5 \%$ in NICM and $57 \%$ in ICM) at 1-year follow-up were lower compared to our results (66.7\% and $73.7 \%$; respectively). Although different study population might have impacted on the discrepancy of the results, it is reasonable to assume that ZF approach does not compromise procedural efficacy. Furthermore, Tanawuttivat et al. reported that CF guided CA acute success rate varied from 76 to $100 \%$, RR was $0-23 \%$ and $C R$ was $0-3 \%$ in 815 patients with idiopathic VAs originating from outflow tracts [27]. In line with these observations, our CA success rate in VAs originating from RVOT was $100 \%$, with $0 \%$ RR and $3.4 \%$ $\mathrm{CR}$. In addition, ZF approach in our study yielded similar OSR $(89.7 \%$ in $\mathrm{SNH}, 82.6 \%$ in ICM, and $62.5 \%$ in NICM patients; $p<0.001$ ) compared to the CF guided CA of VAs reported by Kumar et al. [28] (OSR 79\%, 56\%, and 60\%, respectively; $p<0.001)$ where patients with and without SHD were compared. These observations further affirm the feasibility of ZF approach in various patient groups with VAs.

It has to be recognised that data on completely ZF approaches for CA for VAs is relatively scarce. In some studies, involving a small number of patients with VAs $(\leq 20)$, it has been shown that all CA procedures have been successfully completed with ZF approach and no complications $[12,13]$. In their single-centre experience with a completely ZF approach using the 3D EAM system and ICE in patients with idiopathic VAs, Lamberti et al. [29] and Sanchez et al. [12] determined similar findings regarding outcome and safety. All procedures could be completed without fluoroscopy and reasonable TPT values (respectively; $150 \pm 45 \mathrm{~min}$ and $170.2 \pm 45.7 \mathrm{~min}$ ). Additionally, Razminia et al. [13] reported successful completion of ZF CA in 10 patients with ICM related VTs. Similar to our results, they also remarked that median TPT was $257.5 \mathrm{~min}$ and RR was $21.4 \%$. Moreover, authors of some large studies including patients with different types of arrhythmias, predominantly SVTs, and very few patients with VAs, reported that the use of the ZF approach was possible in $20-100 \%$ of procedures, with an ASR of $80-100 \%$, with a TPT of $128 \pm 21 \mathrm{~min}-$ $169 \pm 43.2 \mathrm{~min}$, and CR of $0-2.5 \%[11,14,30,31]$. These studies based on single experienced operators or singlecentre reports do not have enough statistical power to enable analysis of factors related to the efficacy and safety of ZF CA. Also, the discrepancies between all study results, including ours, can be explained, at least in part, by differences in study design, patient population, VA substrate location, 3D EAM system utilisation, and in follow-up duration.

In a recent large-scale study comparing CF and ZF approach in patients with idiopathic VAs [19] it was implied that all idiopathic VAs cannot be successfully and safely treated completely without the use of fluoroscopy. In this prospective multi-centre study Wang et al. enrolled 489 patients with idiopathic VAs and reported that there was no significant difference between the ZF approach $(\mathrm{n}=163)$ and CF approach $(\mathrm{n}=326)$, including ASR ( $84.1 \% \mathrm{vs} 85.4 \%$, respectively), RR $(1.9 \%$ vs $2.2 \%)$, or severe CR $(0.6 \%$ vs $0.9 \%)$. In line 

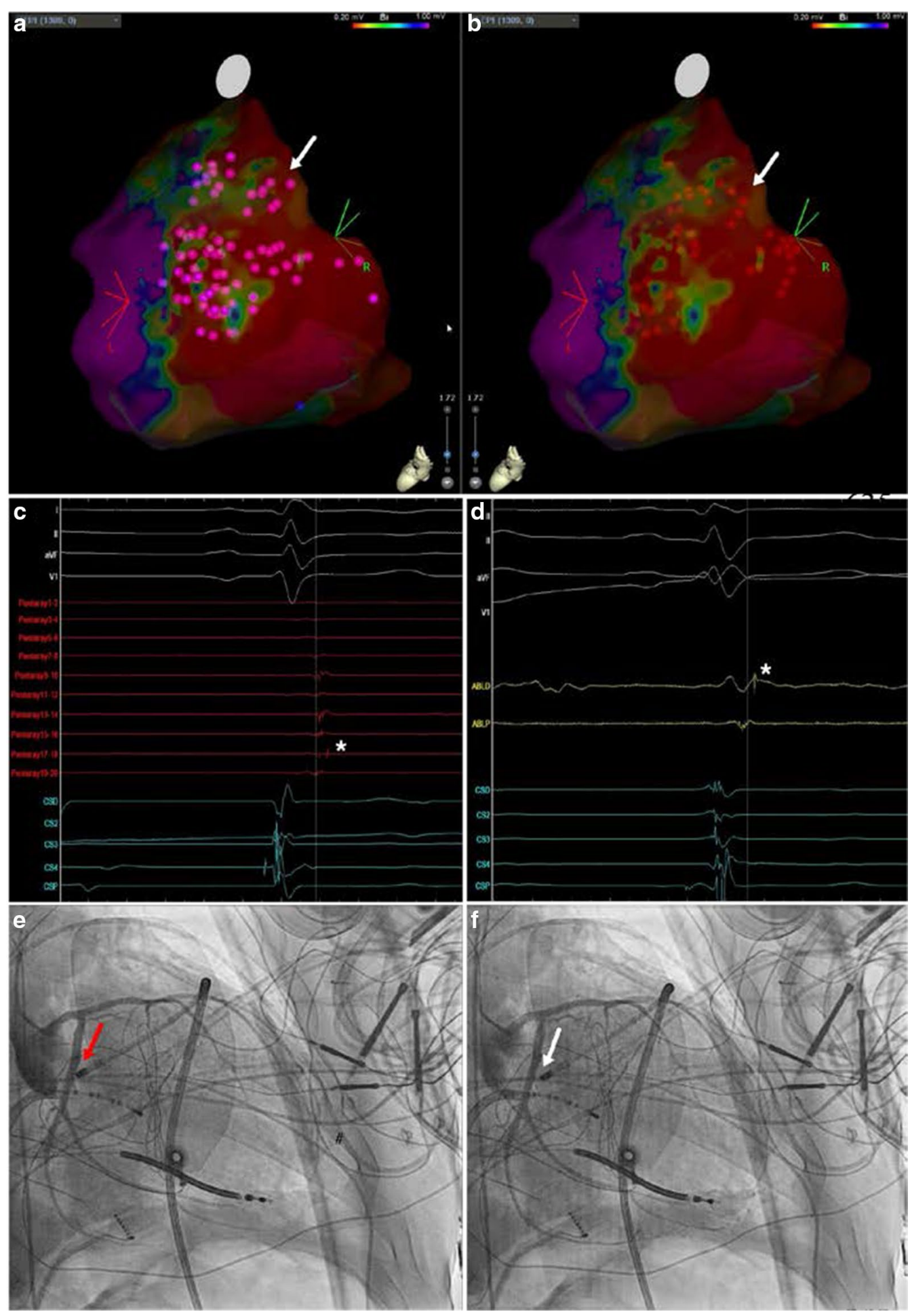

with our observations, there were 9 cases where fluoroscopy was used for coronary angiography. Although it is the largest study on the subject, it is not randomised and, more importantly, excluded patients with SHD.
Additionally, follow-up to determine the arrhythmic recurrence was shorter $(5.4 \pm 3.9$ months $)$ compared to our study. In a study that enrolled patients with SHD (22 ICM and 19 NICM patients), Cano et al. suggested 

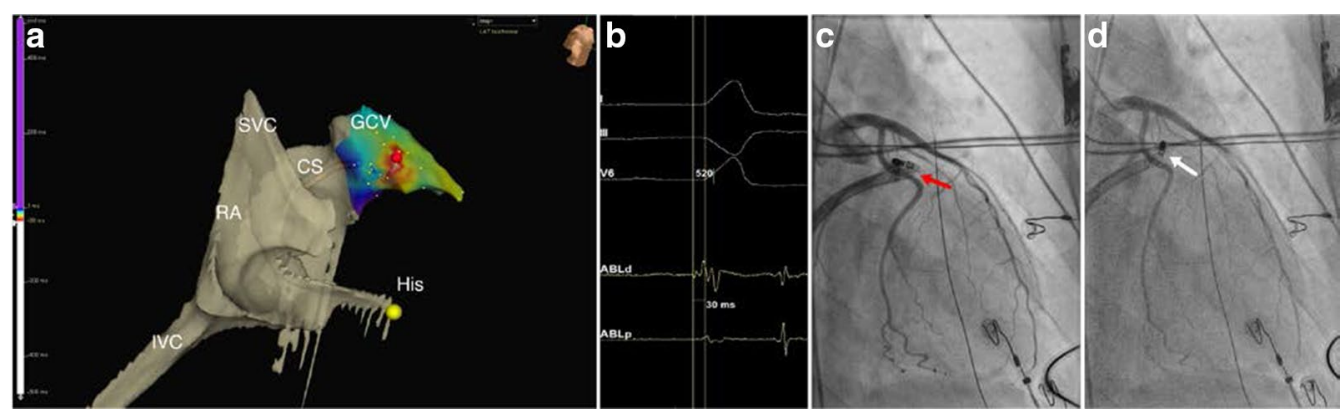

Fig. 2 Left ventricular summit area ablation. (A) A modified right anterior oblique (RAO) view of a partial three-dimensional (3D) electro-anatomical mapping (EAM) activation map of the right atrium (RA), the coronary sinus (CS) and its continuation into the great cardiac vein (GCV) where the earliest local ventricular activation during left ventricular (LV) summit ventricular ectopy was found and successfully ablated (red dot). (B) Surface (I, III and V6) and intra-cardiac electrograms (ABLd and ABLP) revealing the earliest local ventricular activation (- 30 ms) during LV summit ectopy as recorded in the GCV. Before actual ablation at the earliest activation site selective left coronary angiography in a modified RAO view was performed as shown on $(\mathbf{C}, \mathbf{D})$. Red arrow on $(\mathbf{C})$ points at the tip of the ablation catheter touching the proximal part of the LCX in which case the catheter was slightly withdrawn to the site marked with the white arrow on (D) showing the tip at the safe distance from the LCX at the location of the eventual successful ablation. His marks the His bundle location; SVC superior vena cava; IVC inferior vena cava

Table 3 Procedural complications

\begin{tabular}{llll}
\hline & $\begin{array}{l}\text { All } \\
(\mathbf{n = 6 0 )}\end{array}$ & $\begin{array}{l}\text { Group 1 } \\
(\mathbf{n = 3 1 )}\end{array}$ & $\begin{array}{l}\text { Group 2 } \\
\mathbf{( n = 2 9 )}\end{array}$ \\
\hline Stroke/TIA & 0 & 0 & 0 \\
Pericardial tamponade & $1(1.7)$ & 0 & $1(3.4)$ \\
RV perforation & $1(1.7)$ & $1(3.2)$ & 0 \\
Pneumothorax & 0 & 0 & 0 \\
Hematothorax & 0 & 0 & 0 \\
PSA surgery/intervention & $1(1.7)$ & $1(3.2)$ & 0 \\
CIED lead dislocation & 0 & 0 & 0 \\
Overall & $3(5)$ & $2(6.4)$ & $1(3.4)$ \\
\hline
\end{tabular}

TIA transient ischaemic attack, $R V$ right ventricular, $P S A$ arterial pseudoaneurysm, CIED cardiac implantable electronic device

that a significant (but not complete) reduction in radiation exposure can be achieved by using the CARTOUNIVU module in scar-related VT ablation procedures [20]. Median fluoroscopy time in the study population was $6.1 \mathrm{~min}$ and DAP $10.8 \mathrm{Gycm}^{2}$, with higher reported radiation exposures in NICM patients and in epicardial procedures. Compared to our study, completely ZF procedures were not performed and ASR in patients with SHD was somewhat lower (66\% vs.77\%, respectively). However, it has to be recognised that the advantage of the CARTOUNIVU module seems to be in integration of coronary angiography images with the 3D EAM system, thus lowering the fluoroscopy time and DAP in cases where use of X-ray cannot be completely avoided.

The characteristics of the main studies used for discussion purposes are presented along with our findings in the Table 4.

\section{Clinical implications}

Our study shows that completely ZF approach to CA is feasible and safe in most types of VAs even when SHD is present. Furthermore, procedural outcomes of $\mathrm{ZF}$ approach are not inferior to the CF-based procedures. However, to achieve these comparable results reliance on the available ZF oriented technology, namely ICE and available 3D EAM systems, is mandatory. We routinely used ICE in all ablation procedures which possibly contributed to our low CR and relatively high OSR with short TPT. Utilisation of ICE seems to eliminate the need for X-ray fluoroscopy by allowing real-time imaging of catheters during mapping and ablation, monitoring of possible procedural complications (especially pericardial effusion) and direct imaging of anatomical variations and structures (i.e. papillary muscles) that cannot be adequately imaged with the 3D EAM systems [32].

Our results appreciate the inherent limitations of the ZF approach in treatment of VAs. With primarily using 3D EAM system and ICE, there seem to be two major limitations, where the use of fluoroscopy cannot be avoided:

1. Epicardial mapping and ablation and the need to visualise wires, long sheaths and catheters in the epicardial space.

2. Ablation of VAs originating in the LV summit where visualisation of coronary anatomy in necessary to avoid RF delivery close or on top of a large caliber coronary artery. 


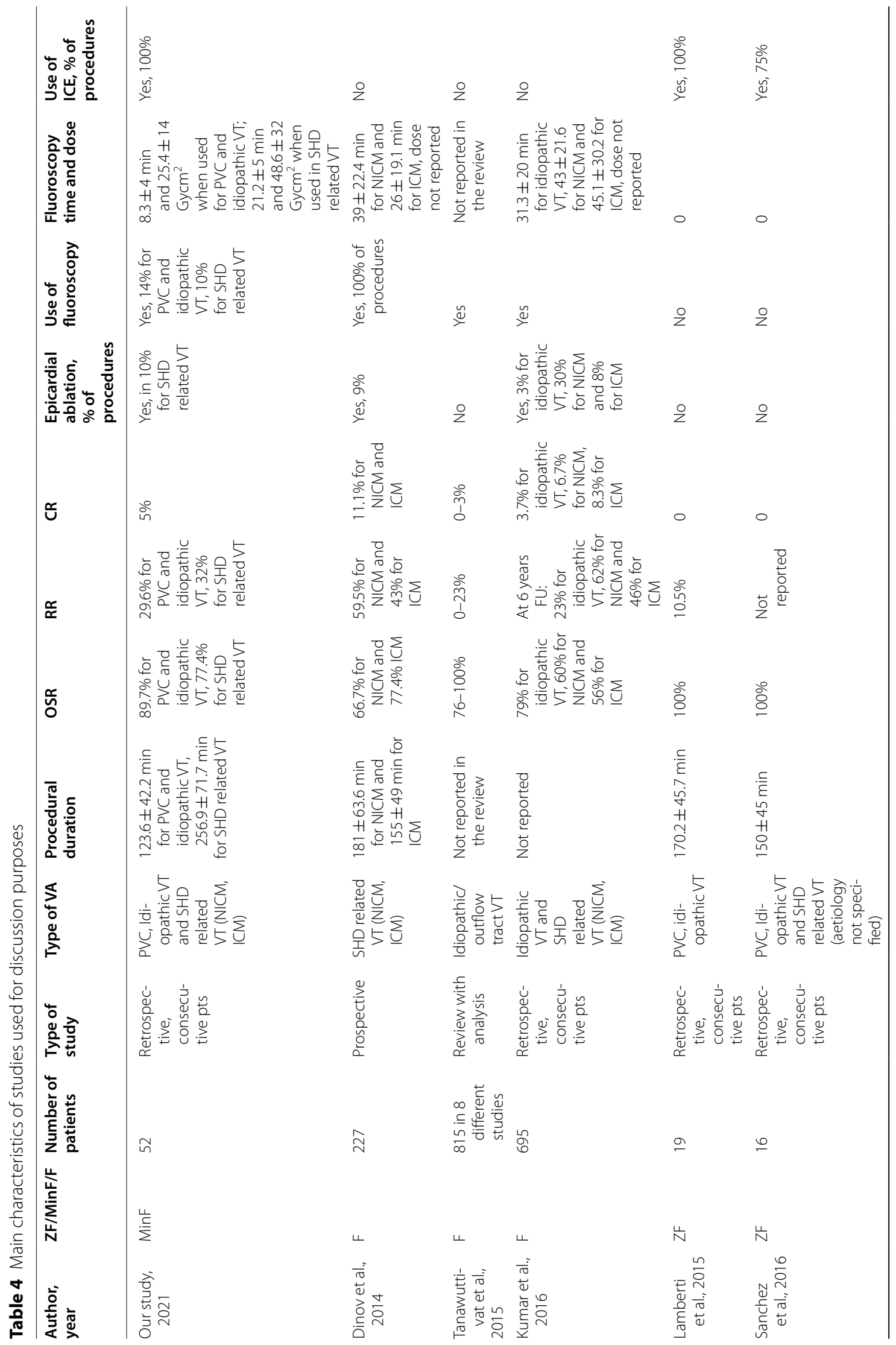




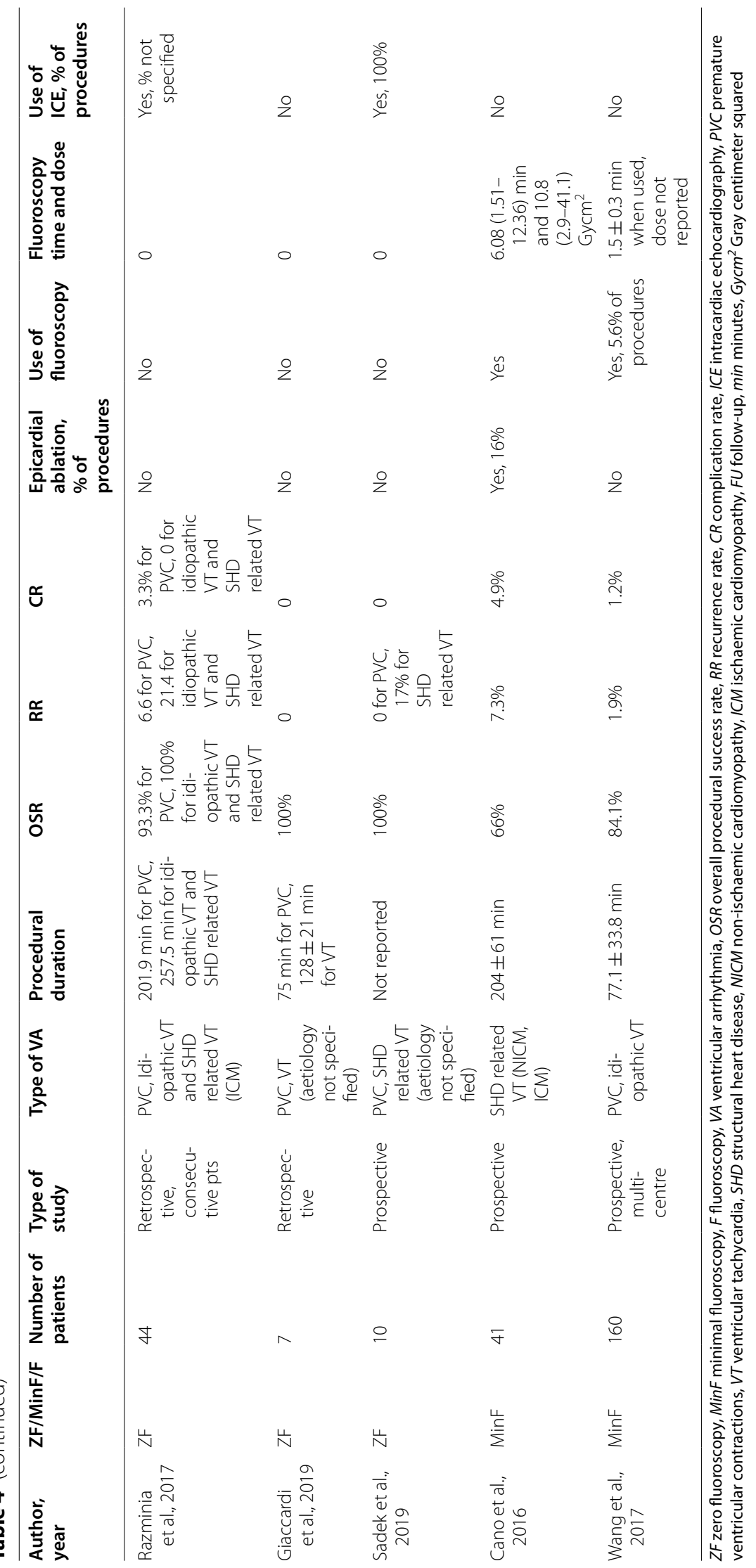




\section{Study limitations}

There are some study limitations decreasing the value of our conclusions. Firstly, this study is observational without a control group and it is not randomised to compare the CF and ZF approaches. Secondly, all procedures were performed by a single operator, already experienced in fluoroless CA of different tachycardias. On the other hand, the fact that a single operator has done all the procedures can be considered a partial advantage in avoiding inter-operator variability. Thirdly, the follow-up period for the detection of possible recurrences of arrhythmias was relatively short, albeit comparable to the available published data. It is reasonable to assume that with a longer follow-up more patients would have experienced arrhythmia recurrences. Fourthly, relatively small study population precluded detailed analysis of VA subgroups, which limits the value of our analysis. Finally, several patient-related factors, namely metabolic and inflammatory status, could affect the electrophysiological properties of the myocardium and outcomes of ablation procedures $[33,34]$. However, a relatively small study population precluded detailed analysis of the potential impact of these factors on procedural outcomes in different VA subgroups.

\section{Conclusions}

Fluoroscopy minimising approach for CA of VAs is feasible and safe in patients with SHD and SNH. Although ZF approach relying only on 3D EAM system and ICE can be utilised in most cases, fluoroscopy could not be completely abolished in VAs with epicardial and LV summit substrate location.

\footnotetext{
Abbreviations

CA: Catheter ablation; CF: Conventional fluoroscopy; VA: Ventricular arrhythmia; AF: Atrial fibrillation; ALARA: As low as reasonably achievable; NZF: Near-zero fluoroscopy; ZF: Zero fluoroscopy; 3D: Three-dimensional; EAM: Electro-anatomical mapping; SVT: Supra-ventricular arrhythmia; SNH: Structurally normal heart; SHD: Structural heart disease; ECG: Electrocardiogram; AAD: Anti-arrhythmic drug; CIED: Cardiac implantable electronic device; CT: Computed tomography; MRI: Magnetic resonance imaging; USA: United States of America; ICE: Intra-cardiac echocardiography; RF: Radio-frequency; LAVA: Local abnormal ventricular activity; LP: Late potential; RV: Right ventricle; VT: Ventricular tachycardia; W: Watt; LV: Left ventricle; DAP: Dose area product; TSP: Trans-septal puncture; TPT: Total procedural time; OSR: Overall procedural success rate; RR: Recurrence rate; CR: Complication rate; ATP: Anti-tachycardia pacing; NY: New York; SD: Standard deviation; ICM: Ischaemic cardiomyopathy; NICM: Non-ischaemic cardiomyopathy; IHD: Ischaemic heart disease; RVOT: Right outflow tract; LCX: Left circumflex coronary artery; RAO: Right anterior oblique; RA: Right atrium; CS: Coronary sinus; GCV: Great cardiac vein; SVC: Superior caval vein; IVC: Inferior caval vein; LVEF: Left ventricular ejection fraction; LVEDV: Left ventricular end-diastolic volume.
}

\section{Acknowledgements}

Not applicable.

\section{Authors' contributions}

All authors (MJ, DŽ, TPK, DK, PT, TK, JK, MR, MY) made substantial contribution to the design of the study, acquisition, analysis and interpretation of data. All authors (MJ, DŽ, TPK, DK, PT, TK, JK, MR, MY) also actively participated in drafting the manuscript, its final revisions and corrections. All authors (MJ, DŽ, TPK, DK, PT, TK, JK, MR, MY) read and approved the final version of the manuscript. All authors (MJ, DŽ, TPK, DK, PT, TK, JK, MR, MY) agree to be personally accountable for their personal contributions to the manuscript.

\section{Funding}

None.

\section{Availability of data and materials}

Data generated or analysed during this study is for the most part included in this published article. Additional raw data is available and can be shared upon request to the corresponding author.

\section{Declarations}

Ethics approval and consent to participate

The study complies with the Declaration of Helsinki. The fluoroless catheter ablation approach was approved by The National Medical Ethics Committee (Komisija za medicinsko etiko Republike Slovenije). All patients (or their parents in case of paediatric patients) gave written informed consent before ablation procedures were performed.

\section{Consent for publication}

Not applicable.

\section{Competing interests}

Matevž Jan received honoraria from Biosense Webster. Other authors report no competing interests.

\section{Author details}

${ }^{1}$ Cardiovascular Surgery Department, University Medical Centre Ljubljana, Zaloška 7, 1000 Ljubljana, Slovenia. ${ }^{2}$ Cardiology Department, University Medical Centre Ljubljana, Ljubljana, Slovenia. ${ }^{3}$ Clinical Institute for Radiology, University Medical Centre Ljubljana, Ljubljana, Slovenia.

Received: 7 February 2021 Accepted: 10 June 2021

Published online: 16 June 2021

References

1. Al-Khatib SM, Stevenson WG, Ackerman MJ, Bryant WJ, Callans DJ, Curtis $A B$, et al. 2017 AHA/ACC/HRS guideline for management of patients with ventricular arrhythmias and the prevention of sudden cardiac death: Executive summary: A Report of the American College of Cardiology/ American Heart Association Task Force on Clinical Practice Guidelines and the Heart Rhythm Society. Heart Rhythm. 2018;15:e190-252.

2. Ait-Ali L, Andreassi MG, Foffa I, Spadoni I, Vano E, Picano E. Cumulative patient effective dose and acute radiation-induced chromosomal DNA damage in children with congenital heart disease. Heart. 2010;96:269-74.

3. Kovoor P, Ricciardello M, Collins L, Uther JB, Ross DL. Risk to patients from radiation associated with radiofrequency ablation for supraventricular tachycardia. Circulation. 1998;98:1534-40.

4. Picano E, Vañó E, Rehani MM, Cuocolo A, Mont L, Bodi V, et al. The appropriate and justified use of medical radiation in cardiovascular imaging: a position document of the ESC Associations of Cardiovascular Imaging, Percutaneous Cardiovascular Interventions and Electrophysiology. Eur Heart J. 2014;35:665-72.

5. Lickfett L, Mahesh M, Vasamreddy C, Bradley D, Jayam V, Eldadah Z, et al. Radiation exposure during catheter ablation of atrial fibrillation. Circulation. 2004;110:3003-10.

6. Clay MA, Campbell RM, Strieper M, Frias PA, Stevens M, Mahle WT. Longterm risk of fatal malignancy following pediatric radiofrequency ablation. Am J Cardiol. 2008;102:913-5.

7. Ector J, Dragusin O, Adriaenssens B, Huybrechts W, Willems R, Ector $\mathrm{H}$, et al. Obesity is a major determinant of radiation dose in patients 
undergoing pulmonary vein isolation for atrial fibrillation. J Am Coll Cardiol. 2007;50(3):234-42.

8. Limacher MC, Douglas PS, Germano G, Laskey WK, Lindsay BD, McKetty $\mathrm{MH}$, et al. ACC expert consensus document. Radiation safety in the practice of cardiology. American College of Cardiology. J Am Coll Cardiol. 1998;31(4):892-913.

9. Jan M, Žižek D, Rupar K, Mazić U, Kuhelj D, Lakič N, et al. Fluoroless catheter ablation of various right and left sided supra-ventricular tachycardias in children and adolescents. Int J Cardiovasc Imaging. 2016;32(11):1609-16.

10. Jan M, Yazici M, Kalinšek TP, Žižek D, Kuhelj D, Pernat A, et al. Fluoroless radiofrequency and cryo-ablation of atrioventricular nodal reentry tachycardia in adults and children: a single-center experience. J Interv Card Electrophysiol. 2020. https://doi.org/10.1007/s10840-020-00791-1.

11. Tuzcu V. Significant reduction of fluoroscopy in pediatric catheter ablation procedures: long-term experience from a single center. Pacing Clin Electrophysiol. 2012;35:1067-73.

12. Sanchez JM, Yanics MA, Wilson P, Doshi A, Kurian T, Pieper S. Fluoroless catheter ablation in adults: a single center experience. J Interv Card Electrophysiol. 2016:45:199-207.

13. Razminia M, Willoughby MC, Demo H, Keshmiri H, Wang T, D'Silva OJ, et al. Fluoroless catheter ablation of cardiac arrhythmias: a 5-year experience. Pacing Clin Electrophysiol. 2017;40(4):425-33.

14. Giaccardi M, Mascia G, Paoletti Perini A, Giomi A, Cartei S, Milli M. Longterm outcomes after "zero X-ray" arrhythmia ablation. J Interv Card Electrophysiol. 2019;54:43-8.

15. Sadek MM, Ramirez FD, Nery PB, Golian M, Redpath CJ, Nair GM, et al. Completely nonfluoroscopic catheter ablation of left atrial arrhythmias and ventricular tachycardia. J Cardiovasc Electrophysiol. 2019;30(1):78-88.

16. Jan M, Žižek D, Kuhelj D, Lakič N, Prolič Kalinšek T, Štublar J, et al. Combined use of electro-anatomic mapping system and intracardiac echocardiography to achieve zero-fluoroscopy catheter ablation for treatment of paroxysmal atrial fibrillation: a single centre experience. Int J Cardiovasc Imaging. 2020;36(3):415-22.

17. Reddy VY, Morales G, Ahmed H, Neuzil P, Dukkipati S, Kim S, et al. Catheter ablation of atrial fibrillation without the use of fluoroscopy. Heart Rhythm. 2010;7(11):1644-53.

18. Huo Y, Christoph M, Forkmann M, Pohl M, Mayer J, Salmas J, et al. Reduction of radiation exposure during atrial fibrillation ablation using a novel fluoroscopy image integrated 3 -dimensional electroanatomic mapping system: A prospective, randomized, single-blind, and controlled study. Heart Rhythm. 2015;12(9):1945-55.

19. Wang Y, Chen GZ, Yao Y, Bai Y, Chu HM, Ma KZ, et al. Ablation of idiopathic ventricular arrhythmia using zero-fluoroscopy approach with equivalent efficacy and less fatigue: a multicenter comparative study. Medicine (Baltimore). 2017;96(6):e6080.

20. Cano Ó, Andrés A, Osca J, Alonso P, Sancho-Tello MJ, Olagüe J, et al. Safety and feasibility of a minimally fluoroscopic approach for ventricular tachycardia ablation in patients with structural heart disease: influence of the ventricular tachycardia substrate. Circ Arrhythm Electrophysiol. 2016;9(2):e003706.

21. Akdeniz C, Gul EE, Celik N, Karacan M, Tuzcu V. Catheter ablation of idiopathic right ventricular arrhythmias in children with limited fluoroscopy. J Interv Card Electrophysiol. 2016;46:355-60.
22. Jaïs $P$, Maury $P$, Khairy $P$, Sacher F, Nault I, Komatsu Y, et al. Elimination of local abnormal ventricular activities: a new end point for substrate modification in patients with scar-related ventricular tachycardia. Circulation. 2012;125(18):2184-96.

23. Miller JM, Tyson GS, Hargrove WC III, Vassallo JA, Rosenthal ME, Josephson ME. Effect of subendocardial resection on sinus rhythm endocardial electrogram abnormalities. Circulation. 1995;91:2385-91.

24. Jackson N, Gizurarson S, Viswanathan K, King B, Massé S, Kusha M, et al. Decrement evoked potential mapping: basis of a mechanistic strategy for ventricular tachycardia ablation. Circ Arrhythm Electrophysiol. 2015;8(6):1433-42.

25. Žižěek D, Antolič B, Prolič Kalinšek T, Štublar J, Kajdič N, Jelenc M, et al. Intracardiac echocardiography-guided transseptal puncture for fluoroless catheter ablation of left-sided tachycardias. J Interv Card Electrophysiol. 2020. https://doi.org/10.1007/s10840-020-00858-z.

26. Dinov B, Fiedler L, Schönbauer R, Bollmann A, Rolf S, Piorkowski C, et al. Outcomes in catheter ablation of ventricular tachycardia in dilated nonischemic cardiomyopathy compared with ischemic cardiomyopathy: results from the Prospective Heart Centre of Leipzig VT (HELP-VT) Study. Circulation. 2014;129:728-36.

27. Tanawuttiwat T, Nazarian S, Calkins $\mathrm{H}$. The role of catheter ablation in the management of ventricular tachycardia. Eur Heart J. 2016;37:594-609.

28. Kumar S, Romero J, Mehta NK, Fujii A, Kapur S, Baldinger SH, et al. Long-term outcomes after catheter ablation of ventricular tachycardia in patients with and without structural heart disease. Heart Rhythm. 2016;13:1957-63.

29. Lamberti F, Di Clemente F, Remoli R, Bellini C, De Santis A, Mercurio M, et al. Catheter ablation of idiopathic ventricular tachycardia without the use of fluoroscopy. Int J Cardiol. 2015;190:338-43.

30. Wannagat S, Loehr L, Lask S, Völk K, Karaköse T, Özcelik C, et al. Implementation of a near-zero fluoroscopy approach in interventional electrophysiology: impact of operator experience. J Interv Card Electrophysiol. 2018;51(3):215-20.

31. Kozluk E, Rodkiewicz D, Piatkowska A, Opolski G. Safety and efficacy of cryoablation without the use of fluoroscopy. Cardiol J. 2018;25:327-32.

32. Demo H, Willoughby C, Jazayeri MA, Razminia M. Fluoroless catheter ablation of cardiac arrhythmias. Card Electrophysiol Clin. 2019;11:719-29.

33. Sardu C, Santamaria M, Funaro S, Sacra C, Barbieri M, Paolisso P. Cardiac electrophysiological alterations and clinical response in cardiac resynchronization therapy with a defibrillator treated patients affected by metabolic syndrome. Medicine (Baltimore). 2017;96(14):e6558.

34. Sardu C, Carreras G, Katsanos S, Kamperidis V, Pace MC, Passavanti MB. Metabolic syndrome is associated with a poor outcome in patients affected by outflow tract premature ventricular contractions treated by catheter ablation. BMC Cardiovasc Disord. 2014;14:176.

\section{Publisher's Note}

Springer Nature remains neutral with regard to jurisdictional claims in published maps and institutional affiliations.

\footnotetext{
Ready to submit your research? Choose BMC and benefit from:

- fast, convenient online submission

- thorough peer review by experienced researchers in your field

- rapid publication on acceptance

- support for research data, including large and complex data types

- gold Open Access which fosters wider collaboration and increased citations

- maximum visibility for your research: over $100 \mathrm{M}$ website views per year
}

At BMC, research is always in progress.

Learn more biomedcentral.com/submissions 